\title{
Clinical and Biochemical Assessment of Maintenance Treatment in Chronic Recurrent Seborrheic Dermatitis: Randomized Controlled Study
}

\author{
Virginie Turlier • Cécile Viode • Elisabeth Durbise • Adeline Bacquey • Ophélie LeJeune \\ Rui Oliveira Soares $\cdot$ Christophe Lauze $\cdot$ Cécile Villeneuve $\cdot$ Amandine Rouquier \\ Christiane Casas · Daniel Redoules • Valérie Mengeaud • Anne-Marie Schmitt
}

To view enhanced content go to www.dermtherapy-open.com Received: December 19, 2013 / Published online: March 19, 2014

(c) The Author(s) 2014. This article is published with open access at Springerlink.com

\section{ABSTRACT}

Introduction: Few studies have investigated the long-term effects of a maintenance regimen in the prevention of relapses in scalp seborrheic dermatitis (SD), in particular following biomarker changes.

Materials and methods: A new shampoo containing beta-glycyrrhetinic acid (18ßGA) in addition to cyclopiroxolamine (CPO) and zinc pyrithione (ZP) was tested in 67 subjects

Trial Registration: Clinical Trials Lisbon Hospital: 0354/11, \#RV3438D2011162.

Electronic supplementary material The online version of this article (doi:10.1007/s13555-014-0047-0) contains supplementary material, which is available to authorized users.

V. Turlier $(\bowtie) \cdot$ A. Rouquier · V. Mengeaud Service Exploration Clinique, Hôtel Dieu,

Toulouse, France

e-mail: virginie.turlier@pierre-fabre.com

C. Viode · O. LeJeune · C. Villeneuve · C. Casas Service de Biologie Clinique, Hôtel Dieu,

Toulouse, France

E. Durbise

Cahors, France

A. Bacquey

Service Systemes et Methodes, Hôtel Dieu,

Toulouse, France suffering from SD with moderate to severe erythema and itching in a biphasic study. After a first common intensive treatment phase (investigational product thrice a week $\times 2$ weeks), subjects randomly received the investigational product once a week $\times 8$ weeks (maintenance) or a neutral shampoo (discontinuation) in a comparative, parallel group maintenance phase. Efficacy was assessed clinically (overall clinical dandruff score, erythema, overall efficacy, selfevaluation), biochemically and microbiologically by quantitative polymerase chain reaction (qPCR), high performance liquid chromatography (HPLC) or enzyme-linked immunoabsorbent assay (ELISA) analysis of scale samples (Malassezia species (restricta and

R. Oliveira Soares

Hospital Cuf Descobertas-Parque das Nações,

Rua Mario Botas, Lisbon, Portugal

C. Lauze

Pierre Fabre Biométrie, Labège Innopole, France

D. Redoules

Service Pharmacologie Clinique, Hôtel Dieu, Toulouse, France

A.-M. Schmitt

Centre de Recherche sur la Peau, Hôtel Dieu, Toulouse, France 
globosa), cohesion proteins (plakoglobins), inflammation (Interleukin (IL)-8, IL-1RA/IL-1 $\alpha$ ) and pruritus (histamine, cathepsin S) markers). Results: During the intensive treatment phase, SD improved significantly $(p<0.0001)$ with a decrease in clinical signs as well as Malassezia species, cohesion proteins, inflammation and pruritus markers. During the maintenance phase, the improvement persisted in the 'maintenance' group only, with a significant intergroup difference. A consistently positive relationship was found between dandruff, itching, erythema and Malassezia populations, histamine levels and IL-1RA/IL-1 $\alpha$ ratio.

Conclusion: The effectiveness of this maintenance regimen was objectively demonstrated at the clinical, biochemical and microbiological level. Correlations between clinical signs and biomarkers could provide clues to explain the resolution of SD and confirm the interest of biomarkers for SD treatment assessment.

Keywords: Biological markers;

Ciclopiroxolamine; Dandruff; Dermatology; Malassezia; Maintenance; Pruritus; Relapse; Seborrheic dermatitis; Zinc pyrithione; 18ß-glycyrrhetinic acid

\section{INTRODUCTION}

Seborrheic dermatitis (SD) is a chronic dermatosis occurring in about $3-5 \%$ of the adult population [1-3], characterized by dandruff, pruritus and, in some cases, mild to marked erythema, and affecting sebum-rich areas such as the scalp. The pathogenesis of SD is complex and appears to result from pathophysiological interactions between scalp skin, cutaneous microflora, and the cutaneous immune system in the stratum corneum (SC) [4].
In particular, Malassezia yeasts, commonly found as part of the normal skin flora $[5,6]$ but present in higher proportions in the scalp skin of patients with SD, were shown to trigger inflammatory and hyperproliferative epidermal responses with incomplete corneocyte differentiation, leading to an impaired SC barrier [4]. Malassezia species (M. restricta and M. globosa) appear to be the most frequently involved [7-9]. In addition, several biomarkers were shown to be modified in SD and restored by therapeutic intervention, and can be studied by non-invasive sampling: inflammation, hyperproliferation, and skin barrier function $[6,10]$.

Treatment of SD has recently focused on controlling inflammation and reducing the number of Malassezia yeasts. Treatments combining keratolytic agents or topical corticosteroids and antifungals have proven their efficacy [11-13]. The combination of ciclopiroxolamine (CPO) and zinc pyrithione (ZP) has recently been proposed, based on the potentially complementary effect of the two compounds. CPO is a broad-spectrum antifungal with an anti-inflammatory activity by inhibition of prostaglandin and leukotriene synthesis [14, 15]. ZP has both non-specific keratolytic and antifungal activity $[16,17]$ and can normalize the impaired SC ultrastructure observed in SD [18, 19]. A $1.5 \% \mathrm{CPO} / 1 \% \mathrm{ZP}$ shampoo displayed a synergistic inhibitory and fungicidal effect on two species of Malassezia (M. globosa and M. restricta) in vitro with greater efficacy than $2 \%$ ketoconazole [20], and highly significantly reduced the extent and severity of scaling, as well as erythema and pruritus, as shown by a randomized clinical study with blinded investigators [21]. Recently, 18 $\beta$-glycyrrhetinic acid (18ßGA) isolated from licorice root was shown to reduce tumor necrosis factor (TNF)- $\alpha$ production and nuclear factor kappa-light-chainenhancer of activated B cells (NF- $\mathrm{kB}$ ) activation 
based on mammalian polymerase inhibition tests and in vivo experiments in a mouse model [22], as well as the expression of pro-inflammatory genes through the inhibition of $\mathrm{NF}-\kappa \mathrm{B}$ and phosphoinositide 3-kinase (PI3K) activity [23]. On the basis of these results, 18ßGA has been suggested as a potential treatment of inflammatory-mediated diseases [23].

On the grounds of the proven individual effect of CPO/ZP and 18ßGA, a shampoo containing these three compounds might therefore be of value in the short-term and long-term treatment of SD.

The purpose of this study was therefore to assess the antidandruff, antifungal and antiinflammatory effect of short-term intensive treatment with such a shampoo, the persistence of the effect, and the value of maintenance treatment in the prevention of recurrences. The correlation between clinical dandruff score and Malassezia load was also determined in order to objectively support the usefulness of maintenance therapy with this investigational product.

\section{METHODS}

\section{Study Design}

This two-center, comparative, randomized, open-label clinical study was designed to compare two parallel groups and was conducted from January to March 2012 at the Centre de Recherche sur la Peau [Skin Research Centre] (Toulouse, France) and Hospital Cuf Descobertas (Lisbon, Portugal).

\section{Patient Population}

\section{Inclusion Criteria}

Female or male patients aged between 18 and 60 years, inclusive, with clinically diagnosed scalp SD including scaling, erythema and pruritus, and an overall clinical score assessed by the investigator as $\geq 20$ (according to the method of Squire and Goode [11], with an intensity $\geq 2$ on at least one of the four scalp areas) were recruited by French and Portuguese dermatologists. At least one other episode of scaling (with erythema and itching) described by the subject had occurred within 6 months before inclusion.

\section{Exclusion Criteria}

Patients with the following criteria were not included: pregnancy or breastfeeding, history of allergy to any of the ingredients of the test product (e.g., CPO, ZP, keluamid, 18 $\beta \mathrm{GA}$, etc.) or of hygiene or hair care products, chronic or acute progressive disease liable to interfere with the results of the study, dermatological condition or progressive skin lesion of the scalp, SD requiring concomitant topical corticosteroid treatment.

Systemic treatment (cardiovascular, endocrine, or anti-inflammatory drugs, corticosteroids, antibiotics, antidepressants, antipsychotics, antineoplastics, immunosuppressants), or topical treatments applied to the scalp within 7 days before inclusion in the study and that could interfere with the results of the study, any product applied to the scalp that was potentially effective on scaling conditions or that could limit the effectiveness of the study product within 2 weeks before inclusion: anti-inflammatory drugs, ketoconazole, antidandruff shampoo, antifungal, vinegar rinse, essential oils, etc., were other reasons for exclusion, as were dying, bleaching, perming, smoothing and/or straightening within 2 weeks before inclusion. 


\section{Study Products, Randomization and Blinding}

The investigational product was an antidandruff shampoo (Kelual DS ${ }^{\circledR}$, DUCRAY Dermatological Laboratories, Boulogne Billancourt, France) containing $\mathrm{CPO}, \mathrm{ZP}$ and $18 \beta \mathrm{GA}$ and a washing base, while the "control" product was a neutral shampoo (Extra Doux ${ }^{\circledR}$, Ducray laboratories). Both test and control shampoos were provided by Pierre Fabre Dermo-Cosmétique laboratories. Each use of the investigational product consisted of two applications with a 3-min exposure time each before rinsing. The two products were labeled and numbered according to a computergenerated randomization list established by Pierre Fabre Biometrie and were allocated to each patient according to their order of inclusion.

The study was open-label because the patients could easily differentiate between the test products by virtue of their very different smell, viscosity and color.

\section{Treatment Schedule}

The study took place over 10 weeks, including a 2-week intensive treatment phase and an 8 -week maintenance phase. On the day of inclusion (W0), all patients received the investigational product for use thrice a week $\times 2$ weeks (i.e., six times during the intensive treatment phase), and the last use had to take place 2 days before visits so that the interval since using the shampoo was about 2 days $\left( \pm \frac{1}{2}\right.$ day). Patients could use neutral shampoo between two treatments if they were used to shampooing more than thrice a week, but still observing the interval of 2 days without the use of any shampoo before visits. At the beginning of the maintenance phase (W2), patients were randomly allocated either to continue the treatment with the investigational product once a week and the neutral shampoo additionally in order to achieve the usual shampooing frequency (maintenance group), or to use the neutral shampoo only at their usual frequency (control group) for an additional 8 weeks. This protocol left some degree of freedom to patients in order to encourage compliance.

Any treatment liable to interfere with the evaluation of the study outcome, such as permanent waves and coloring, shampoos not provided in the study, systemic or topical treatments with antibiotics, antifungals, corticosteroids or retinoids, or any topical treatment for SD or dandruff, was strictly forbidden for the whole of the study.

\section{Study Plan}

At each visit, i.e., at inclusion (W0), after week 1 (W1) and 2 (W2) of the intensive treatment phase, and after week 4 (W6) and 8 (W10) of the maintenance phase, the investigator performed a scalp examination and swab sampling on welldefined lesional areas for biochemical and microbiological evaluations, scored clinical parameters (dandruff, erythema), and assessed treatment global efficacy. In the meantime, patients self-assessed global efficacy and their scaling condition (i.e., dandruff, itching, discomfort) using questionnaires.

\section{Study Endpoints}

\section{Primary Endpoint}

The main efficacy endpoint was the antifungal action assessed by measuring the change in $M$. restricta and globosa populations by polymerase chain reaction (PCR) assay at the end of the intensive treatment phase (W2) compared with WO. 


\section{Secondary Endpoints}

To evaluate the persistence of intensive treatment and the value of maintenance therapy for 8 weeks after the end of the intensive treatment phase, several clinical parameters and biochemical and microbiological cutaneous markers of SD were used as secondary endpoints.

\section{Clinical Endpoints}

- Change in overall clinical dandruff score, graded by the investigator, at $\mathrm{W} 1, \mathrm{~W} 2, \mathrm{~W} 6$ and W10 compared with W0. The overall clinical dandruff score, ranging from 0 to 80 , was calculated taking into account the area covered by SD lesions and their severity, as described by Squire and Goode [11].

- Change in irritation symptoms from WO at each visit by rating erythema on a 4-point, semi-quantitative scale: $0=$ absent, $1=$ mild, $2=$ moderate, $3=$ severe .

- Global efficacy assessment by the investigator and the subject at each visit, measured by the SGA (subjective global assessment) score, using a 5-point scale ( $1=$ worse, $\quad 2=$ stabilization $/$ no change, $3=$ slight $\quad$ improvement,$\quad 4=$ significant improvement, $5=$ complete resolution or complete remission).

The same investigator rated overall clinical dandruff score, erythema, global efficacy for the same patient during the study.

- Weekly self-assessment of the scaling condition (dandruff, discomfort, itching) on a 4-point scale $(0=$ absent, $1=$ mild, 2 = moderate, 3 = severe), by means of questionnaires issued to the subjects, and global efficacy using the SGA score.

- Time to first recurrence (when condition $\geq$ baseline score) for at least one of the three items: dandruff, discomfort or itching.
- Compliance (number of uses of the investigational product reported by the subject compared with the theoretical number).

\section{Biochemical and Microbiological Cutaneous Markers of SD}

The change in $M$. restricta and globosa populations at W1, W6 and W10 from W0 was assessed by PCR measurements.

The changes in cohesion proteins [plakoglobins (PLK)] and markers of inflammation [Interleukin (IL)-8, IL-1RA/IL-1 $\alpha$ ] and pruritus (histamine, cathepsin S) were assessed at W2 and W10 versus W0 by measuring protein concentrations from swab samples using enzyme-linked immunoabsorbent assay (ELISA) (DuoSet ${ }^{\circledR}$, R\&D Systems, Minnesota, USA), Western blot and high performance liquid chromatography (HPLC) techniques.

\section{Biochemical and Microbiological \\ Measurements}

Scalp Sampling Samples were taken from four lesional areas of $4 \mathrm{~cm}^{2}$ per subject (initially exhibiting scales and erythema), selected from the whole of the scalp and pinpointed by means of a transparent plastic bonnet so as to be identifiable at each visit. The samples for biological markers were obtained by swabbing the areas of scaling with a cotton swab impregnated with a mixture of phosphate buffered saline (PBS) buffer containing 1\% or $0.1 \%$ Triton X100 except for histamine analysis (water).

Biomarker Analysis The overall load of $M$. restricta and globosa was measured by PCR assays performed on swab samples from localized lesional zones of the scalp after DNA extraction. The Malassezia species were 
Table 1 Specific probes for Malassezia species analysis

\begin{tabular}{lll}
\hline Mala_spp & Forward & CTAAATATCGGGGAGAGACCGA \\
Reverse & GTACTTTTAACTCTCTTTCCAAAGTGCTT \\
Probes & TTCATCTTTCCCTCACGGTAC \\
Fala_globosa & Forward & CGCCAAGCGCGCTCT \\
& Reverse & ATCATCAGGCATAGCATG \\
Pala_restricta & Frobes & GGCGGCCAAGCAGTGTTT \\
& Forward & AACCAAACATTCCTCCTTTAGGTGA \\
& Reverse & TTCTCCTGGCATGGCAT \\
\hline
\end{tabular}

analyzed by absolute PCR quantification with TaqMan $^{\circledR}$ (Life Technologies Ltd, Paisley, Scotland) specific probes, using an ABI 7900HT Fast Real-Time PCR System (Applied Biosciences, Life Technologies Ltd) (Table 1). A reference range for each species (Mala_spp) was used for quantification purpose. All quantities were adjusted to the same surface area.

IL-8, IL1 $\alpha$, IL-1RA and cathepsin $S$ were measured by ELISA using specific kits (DuoSet $^{\circledR}$, ref. DY208, DY200, DY280, KGE0068). Histamine was measured by HPLC as described by Kerr et al. [24].

Total protein concentration was used to standardize the inflammation and pruritus markers.

PLK was measured by Western blotting, using mouse anti-PLK as primary antibody (1/1,000, Zymed Laboratories, Inc. California, USA) and goat anti-mouse horseradish peroxidase (HRP) (1/10,000, Jackson ImmunoResearch Europe Ltd. Suffolk, UK) as secondary antibody. All samples were quantified by densitometry after immunodetection with the enhanced chemiluminescence (ECL) kit (Merck Millipore $^{\mathrm{TM}}$, Massachusetts, USA) and standardized for total keratins.

\section{Safety}

Local tolerance was assessed at each postbaseline visit by the investigator. In the case of an adverse event, its nature, intensity, beginning and end dates, and any relationship with the study treatment were reported to the investigator and recorded. Serious adverse events had to be reported to the sponsor within $24 \mathrm{~h}$ of notification by the investigator.

\section{Statistical Methods}

Statistical analysis was performed using SAS $^{\circledR}$ software (SAS Institute, Version 8.2., Buckinghamshire, UK). All quantitative variables were expressed as sample size, mean, standard deviation (SD), median and range values, and qualitative variables as percentage and frequency. All statistical analyses were performed on the full analysis set (FAS) population, but also on the per protocol (PP) population for the primary endpoint analysis.

Time-effect analysis during the intensive treatment phase was performed using paired Student's $t$ test for changes at W2 versus W0, then either the Kolmogorov-Smirnov test, or Wilcoxon's signed rank test, or Student's $t$ test for changes at W1 versus W0, while changes 
during the maintenance phase (W6/W10 vs. W2) were analyzed using an analysis of covariance (ANCOVA) with treatment as a fixed factor, subject as a random factor and W0 as the covariate.

Comparisons of changes between treatment groups (maintenance phase) were analyzed using the Chi square test.

The correlation analysis was performed using Pearson's correlation test. All statistical tests were performed at a significance level of 0.05 .

All procedures were in accordance with the ethical principles stated in the Declaration of Helsinki of 1975, as revised in 2000 and 2008, in conformity with local legal requirements in each country and after approval of the study protocol by the Lisbon Hospital Ethic Committee. Informed consent was obtained from all patients before being included in the study.

\section{RESULTS}

\section{Study Population}

A total of 67 patients (27 in France and 40 in Portugal) with a mean age of $33.63 \pm 10.21$ years were included, 26 males and 41 females, constituting the FAS population. They were randomized to the treated group $(n=33,9$ males, $32.4 \pm 10.0$ years [19.0-59.0]) and the control group $(n=34,17$ males, $34.9 \pm 10.4$ years $[20.0-53.0])$ at $\mathrm{W} 2$ after the intensive treatment phase. Both groups were clinically comparable at W2. Four subjects withdrew prematurely from the study (two in each center) unrelated to the study product, resulting in a PP population of 63 patients.

\section{Primary Endpoint}

Compared with W0, a highly significant decrease was observed after 2 weeks of treatment: $-97.8 \%$ for Malassezia restricta and $-94.9 \%$ for $M$. globosa $(p<0.0001$ for both species). The time course of the Malassezia restricta and globosa populations after shortterm intensive treatment is shown in Fig. 1.

\section{Secondary Endpoint}

\section{Microbiological Markers}

A highly significant drop in log Malassezia restricta and globosa was observed after 1 week of treatment, i.e., three applications $(p<0.0001$ for all measurements, Fig. 1). Subsequently, compared with the end of the intensive treatment phase (W2), log Malassezia increased throughout the maintenance phase in each group. However, whereas this increase was highly significant in the control group at W6 and W10 $(p<0.0001$ for $\log M$. restricta and globosa), it was only significant for $\log M$. globosa at W10 $(p<0.01)$ in the treated group (Fig. 1). During the maintenance phase, in the control group there was a return to baseline levels at W10 for $\log M$. restricta and W6 and W10 for $\log$ M. globosa ( $p>0.05$ versus W0). Noticeably, a highly significant difference was observed between the two groups at each time point $(p<0.0001)$, Malassezia spp. levels being significantly higher in the control group than in the treated group (Fig. 1).

\section{Clinical Evaluation}

A clinical improvement was observed during the study, with a highly significant decrease in overall clinical dandruff score during the intensive treatment phase and a non-return to baseline conditions in the two groups at W6 and W10, the intergroup difference being highly significant at each time point in favor of the treated group $(p<0.0001$ for the difference in changes at W6 and W10 vs. W2) (Table 2). 


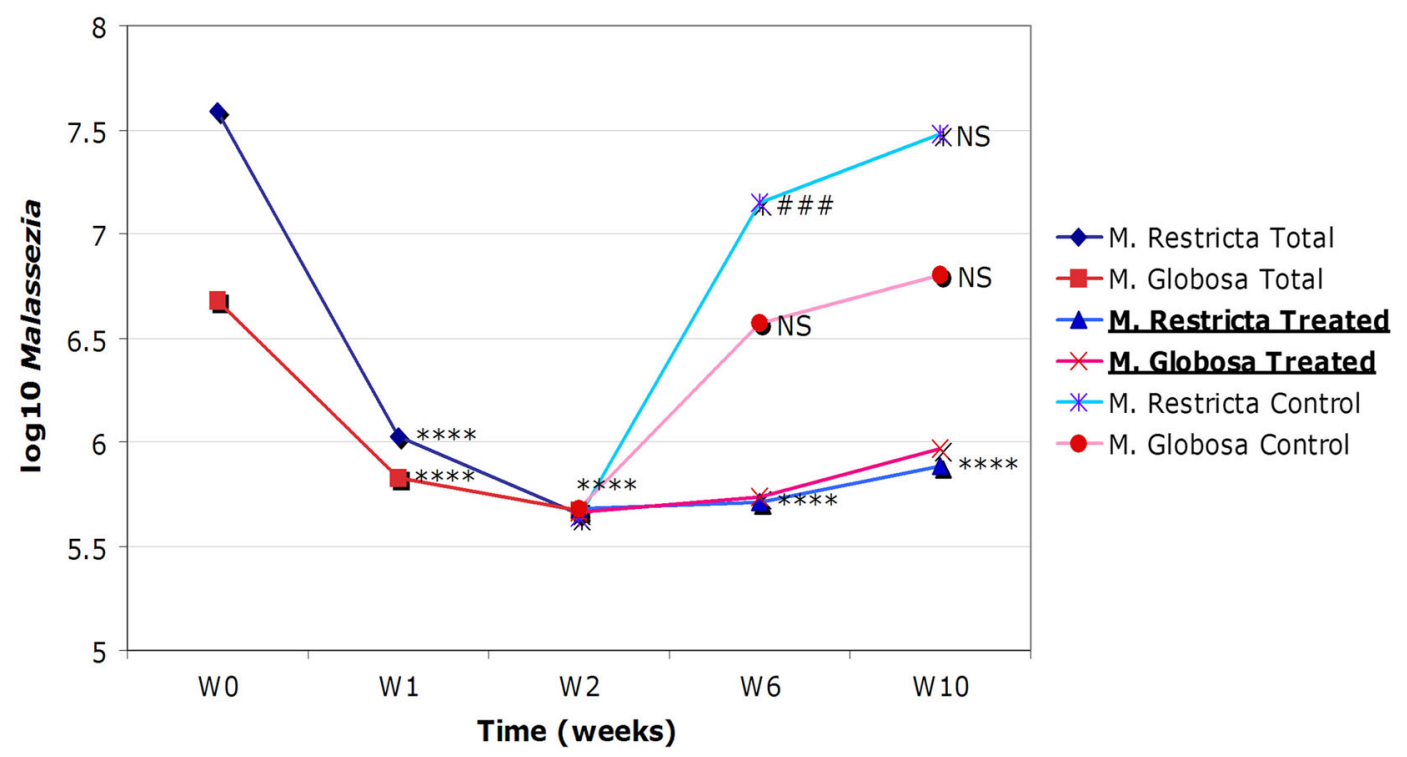

Fig. 1 Time course of $\log 10$ Malassezia restricta and globosa populations during the intensive treatment phase (W0 to W2) in the global study population and during the maintenance phase (W2 to W10) in control and treated

A highly significant decrease in clinical score for erythema at $\mathrm{W} 1$ and $\mathrm{W} 2$ reflected a rapid improvement in irritation during the intensive treatment phase (Fig. 2a), which was maintained at $\mathrm{W} 10$ in the treated group $(p<0.0001$ versus W0) but returned to baseline in the control group ( $p>0.05)$.

The subjective global efficacy (SGA) score assessed by investigator and subject increased during the intensive treatment phase and was maintained (i.e., non-return to baseline in the two groups), especially in the treated group: an investigator-assessed cumulative improvement (i.e., slight and significant improvement and complete recovery) was found in $97 \%$ of cases at $\mathrm{W} 2$, and in $76 \%$ in the treated group versus $27 \%$ in the control group at W10 (Fig. 2b). Likewise, a subject-assessed cumulative improvement was found in $94 \%$ of cases at W2, and in $70 \%$ in the treated group versus $20 \%$ in the control group at W10.

Self-assessment of scaling, discomfort and itching by patients revealed a sustained

patients. Comparisons versus W0 using the KolmogorovSmirnov test. ${ }^{* * * *} p<0.0001 ;{ }^{\# \# \#} p<0.0005 ; N S$ not significant $(p>0.05)$

improvement at $\mathrm{W} 10$ versus $\mathrm{W} 0$ in the treated group compared with a return to baseline in the control group for dandruff $(p<0.0001$ and $p>0.05$ at W10 versus W0 in the treated and control groups, respectively) and discomfort $(p<0.0001$ and $p>0.05$, respectively), whereas itching continued to be improved in both groups $(p<0.0001$ and $p<0.05$, respectively) (Fig. 2c-e). As regards the changes between W0 and W10, the intergroup difference was significant or very significant for discomfort, dandruff, and itching.

Very good compliance was observed, with a significant negative correlation with the $M$. restricta population during the first week of the intensive treatment phase (which decreased when compliance increased, $r=-0.41439$, $p<0.001)$. Time to first recurrence was studied as a survival analysis, as illustrated in Fig. 3, and a log-rank test (Chi squared) showed a statistically significant difference between the two groups in favor of the treated group $(p<0.0001)$. 
Table 2 Mean overall clinical dandruff scores and changes over time during the intensive treatment phase (W0 to W2) in the global study population and during the maintenance phase (W2 to W10) in control and treated patients

\begin{tabular}{|c|c|c|c|}
\hline \multirow[t]{2}{*}{ Clinical parameter } & \multicolumn{3}{|l|}{ Overall clinical score } \\
\hline & $\overline{\text { Treated }(\text { mean } \pm \text { SD })}$ & Control $($ mean \pm SD) & $p$ value $^{\mathrm{s}}$ \\
\hline \multicolumn{4}{|l|}{ Intensive treatment phase } \\
\hline W0 & $30.8 \pm 12.3$ & & \\
\hline W1 & $18.3 \pm 9.6$ & & \\
\hline W2 & $9.6 \pm 8.4$ & & \\
\hline \% Change W1 vs. W0 & $-40.5^{* * * *}$ & & \\
\hline \% Change W2 vs. W0 & $-68.8^{* * * *}$ & & \\
\hline \multicolumn{4}{|l|}{ Maintenance phase } \\
\hline W2 & $9.6 \pm 8.3$ & $9.7 \pm 8.7$ & \\
\hline W6 & $5.9 \pm 6.3$ & $16.3 \pm 11.4$ & \\
\hline \% Change W6 vs. W0 & $-79.9^{* * * *}$ & $-49.2^{* * * *}$ & 0.0407 \\
\hline \% Change W6 vs. W2 & $-37.8^{*}$ & $+68.4^{* * *}$ & $<0.0001$ \\
\hline W10 & $5.15 \pm 6.6$ & $21.60 \pm 12.2$ & \\
\hline \% Change W10 vs. W0 & $-82.6^{* * * *}$ & $-32.5^{* * * *}$ & $<0.0001$ \\
\hline \% Change W10 vs. W2 & $-46.0^{* *}$ & $+123.7^{* * * *}$ & $<0.0001$ \\
\hline
\end{tabular}

Intragroup comparison of mean \pm standard deviation (SD) change versus W0 or W2 using Kolmogorov-Smirnov, Wilcoxon's signed rank or paired Student's $t$ test, ${ }^{*} p<0.05,{ }^{* *} p<0.01,{ }^{* * *} p<0.001,{ }^{* * *} p<0.0001$

$S D$ standard deviation

\$ Intergroup comparison using an analysis of covariance (ANCOVA)

\section{Biochemical Markers}

At the end of the intensive treatment phase, the levels of inflammation and pruritus markers decreased significantly: cathepsin S by $-70 \%$ $(p<0.0001)$, histamine by approximately $-65 \%$ $(p<0.0001)$ (Fig. 4$)$ and IL-8 by $-87.5 \%$ $(p<0.05)$. The IL-1RA/IL- $1 \alpha$ ratio decreased by about $-20 \% \quad(p<0.05)$, reflecting an antiinflammatory process. The cell cohesion marker PLK decreased significantly $(-41.7 \%$, $p<0.01$ ) (Table 3).

During the maintenance phase, the treatment effect regressed significantly in the control group (Table 3) for all markers. In the treated group, the decrease was only reversed in the case of histamine levels, which reverted to the initial state at the end of the maintenance phase and was not statistically different from the control group at W10.

Levels of other markers remained unchanged (cathepsin S, IL-8) or declined slightly in the treated group, especially for IL-1RA/IL- $1 \alpha$, with a significant difference at W10 versus W0 and W2 $(p<0.005$ and $p<0.05$, respectively). There was a significant difference between the two groups for the changes from baseline in IL-8 $(p<0.05)$ and IL-1RA/IL- $1 \alpha$ ratio $(p<0.05)$ in favor of the treated group, with a significantly greater regression of the treatment effect in the control.

PLK levels continued to decline in the treated group $(-18.2 \%, p>0.05$ versus $\mathrm{W} 2)$, 
$\mathbf{a}$

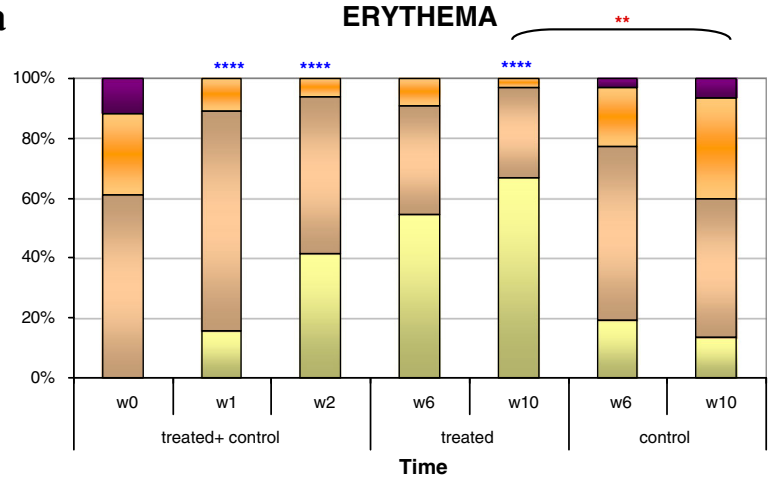

$\square$ Absent $\square$ Mild $\square$ Moderate $\square$ Severe

c

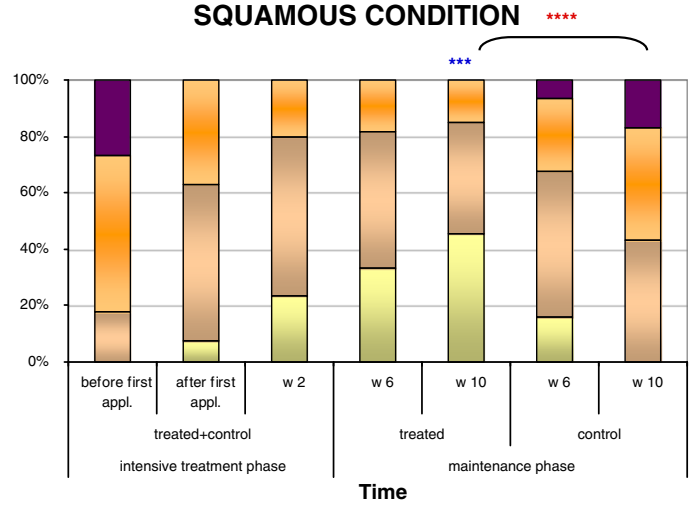

aabsent $\square$ mild $\square$ moderate $\square$ severe

e

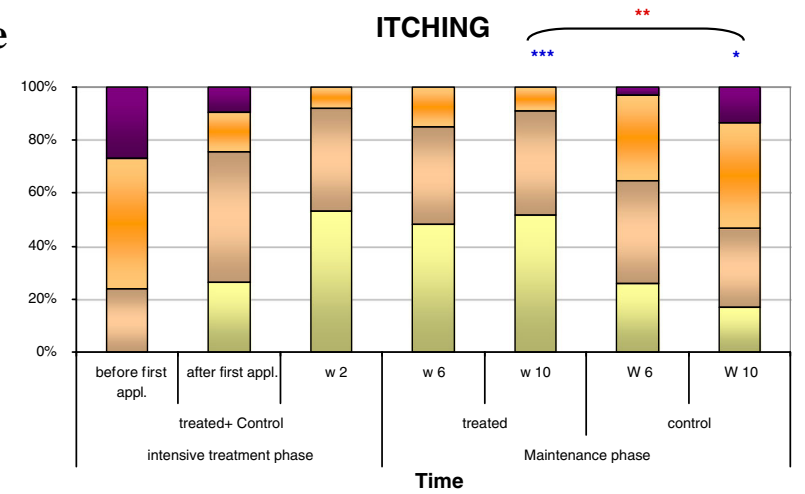

$\square$ absent $\square$ mild घ moderate $\square$ severe

Fig. 2 a-e Evolution of clinical criteria during the intensive treatment phase (W0 to W2) in the global study population and during the maintenance phase (W2 to W10) in control and treated patients (a erythema, b global efficacy, c self-assessment of scaling, d selfassessment of discomfort, e self-assessment of itching). For the intensive treatment phase, a paired Student's $t$ test or a Wilcoxon's signed rank test, depending on normality of

\section{b GLOBAL EFFICACY : INVESTIGATOR'S ASSESSMENT}

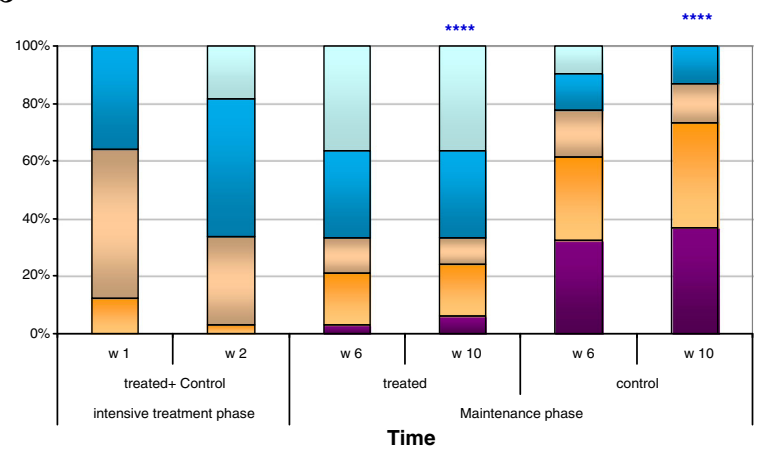

$\square$ worsening $\square$ no change $\square$ slight improvement $\square$ significant improvement $\square$ complete recovery

\section{d}

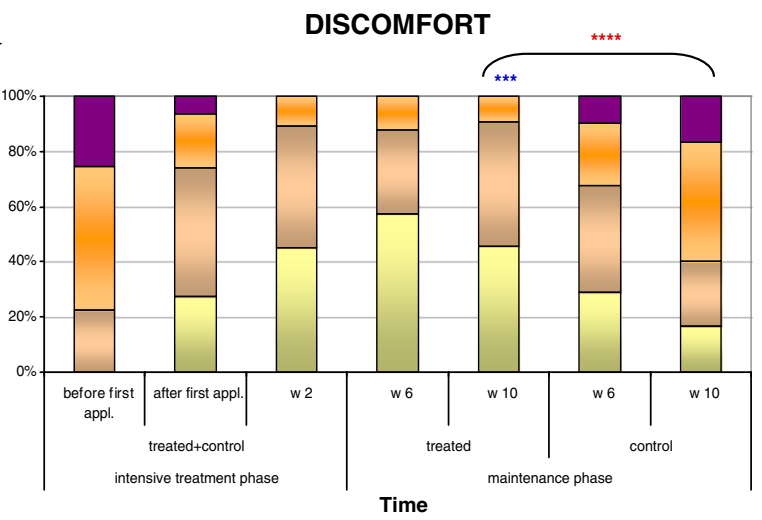

$\square$ absent $\square$ mild $\square$ moderate $\square$ severe

distributions, was used on changes between W0 and W1 and W2, respectively. For the maintenance phase, a Wilcoxon's signed rank test was used on changes between W2 and W6 and W10, respectively. ${ }^{*} p<0.05,{ }^{* *} p<0.01$, ${ }^{* * *} p<0.001,{ }^{* * * *} p<0.0001$, in blue: intragroup versus W0, in red: intergroup W10/W0 


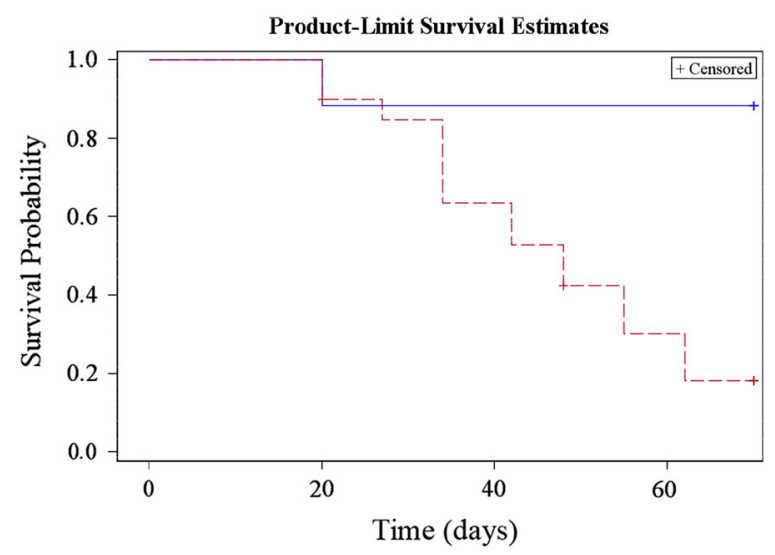

Fig. 3 Time to first recurrence is shown for the treated group (in blue) and control group (in red, dotted line). Logrank test (Chi squared) between the two groups, $p<0.0001$
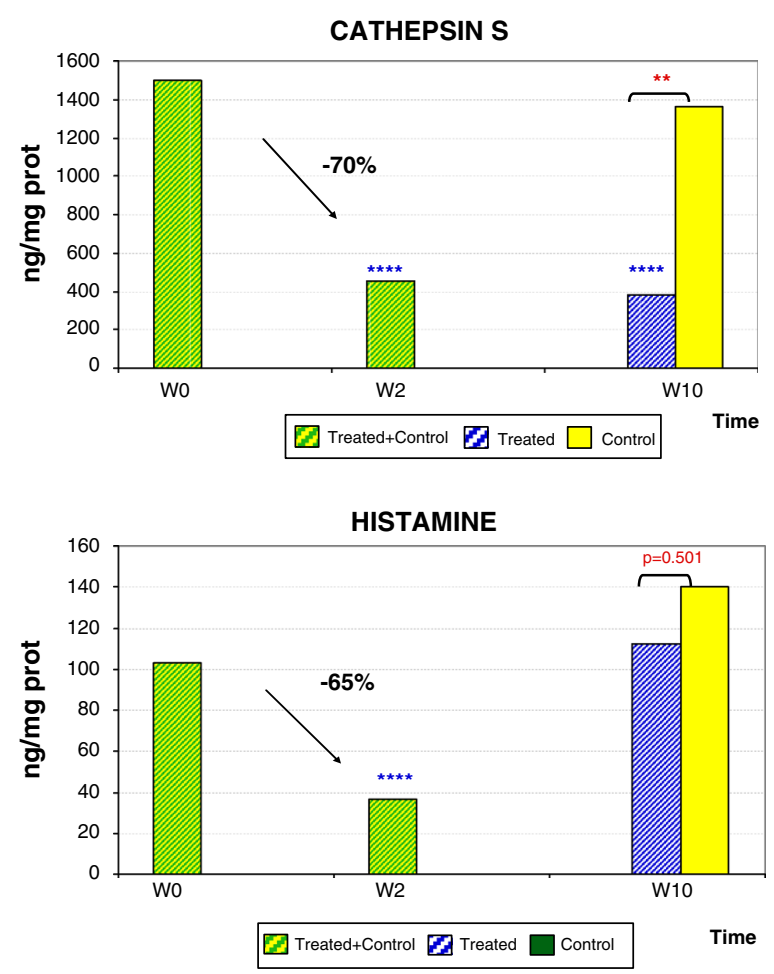

Fig. 4 Pruritus biomarkers during the intensive treatment phase (W0 to W2) in the global study population and during the maintenance phase (W2 to W10) in control and treated patients. ${ }^{* *} p<0.01,{ }^{* * * *} p<0.0001$, in blue: intragroup versus W0, in red: intergroup W10/W0

reflecting an improvement in scaling, whereas it significantly increased in the control group $(+4.2 \%, p<0.005)$ to reach a non-statistically different level at W10 versus baseline. Intergroup comparison showed a significant difference in the time course of PLK during the maintenance phase in favor of the treated group $(p<0.01)$, revealing a maintenance effect in the treated group only.

\section{Correlations}

As shown in Table $4 \mathrm{a}-\mathrm{c}$, the overall clinical score was positively correlated with histamine and $\log$ M. restricta (Table 4a), log M. globosa in the treated group (Table 4c) and IL-1RA/IL1 $\alpha$ in the control group (Table 4c), and during the intensive treatment phase (Table $4 \mathrm{~b}$ ).

Erythema was positively correlated with IL1RA/IL-1 $\alpha$, histamine and PLK in the treated group (Table $4 a)$.

Itching was positively correlated with IL1RA/IL-1 $\alpha$ and $\log$ M. total in the control group (Table 4c), while global efficacy was negatively correlated with histamine in the treated group (Table 4a, c), IL-1RA/IL-1 $\alpha$ (Table $4 b$ ), and log M. total in the control group (Table $4 \mathrm{a}$ ).

\section{Safety}

Twenty-seven adverse events unrelated to the study drug, including one pregnancy (which led to early study termination), and 23 adverse events suspected of being related to the investigational product were identified. These were mainly transient side effects of low to mild severity, such as stinging, itching, and burning sensations, and resolved spontaneously within 1 week without any particular action, resulting in a decrease in the percentage of 'good' tolerance $(27 \%, 5 \%, 3 \%$ and $3 \%$ at week 1,2 , 6 and 10, respectively, in the maintenance group), and an increase in the percentage of 'very good' tolerance with time (73\%, 95\%, 97\% and $97 \%$ at week 1, 2, 6 and 10, respectively, in the maintenance group). 
Table 3 Mean biochemical parameters and changes over time during the intensive treatment phase (W0 to W2) in the global study population and during the maintenance phase (W2 to W10) in control and treated patients

\begin{tabular}{|c|c|c|c|c|c|c|c|}
\hline \multirow{2}{*}{$\begin{array}{l}\text { Biochemical } \\
\text { parameter }\end{array}$} & \multicolumn{3}{|c|}{ Intensive treatment phase } & \multicolumn{4}{|c|}{ Maintenance phase } \\
\hline & $\begin{array}{l}\text { Wo } \\
(\text { mean } \pm \text { SD })\end{array}$ & $\begin{array}{l}\text { W2 } \\
(\text { mean } \pm S D)\end{array}$ & $\begin{array}{l}\% \text { Change } \\
\text { W2 vs. } \\
\text { W0 }\end{array}$ & $\begin{array}{l}\text { W2 } \\
(\text { mean } \pm S D)\end{array}$ & $\begin{array}{l}\text { W10 } \\
(\text { mean } \pm S D)\end{array}$ & $\begin{array}{l}\text { \% Change } \\
\text { W10 vs. } \\
\text { W2 }\end{array}$ & $\begin{array}{l}\text { \% Change } \\
\text { W10 vs. } \\
\text { W0 }\end{array}$ \\
\hline \multicolumn{8}{|c|}{ Histamine ( $\mathrm{ng} / \mathrm{mg}$ proteins) } \\
\hline Treated & $103.2 \pm 188.1$ & $36.4 \pm 87.9$ & $-64.8^{* * * *}$ & $45.3 \pm 115.7$ & $112.4 \pm 185.8$ & $147.8^{*}$ & 1.35 \\
\hline Control & & & & $27.2 \pm 44.3$ & $140.0 \pm 176.7$ & $415.3^{* * *}$ & 46.7 \\
\hline$p$ value $\$$ & & & & & & NS & NS \\
\hline \multicolumn{8}{|c|}{ Cathepsin S (ng/mg proteins) } \\
\hline Treated & $1,500.5 \pm 976.5$ & $450.5 \pm 679.9$ & $-70^{* * * *}$ & $317.8 \pm 471.6$ & $381.1 \pm 529.3$ & 19.9 & $-73.1^{* * * *}$ \\
\hline Control & & & & $592.7 \pm 834.6$ & $1,364.6 \pm 941.7$ & $130.2^{* *}$ & -14 \\
\hline$p$ value $\$$ & & & & & & 0.0062 & 0.0062 \\
\hline \multicolumn{8}{|c|}{ IL-8 (pg/mg proteins) } \\
\hline Treated & $178.3 \pm 529.4$ & $22.3 \pm 79.5$ & $-87.5^{*}$ & $41.3 \pm 111.9$ & 0 & -100 & $-100^{* * * *}$ \\
\hline Control & & & & $8.0 \pm 39.0$ & $47.4 \pm 94.6$ & 495.2 & $-59.2^{* *}$ \\
\hline$p$ value $\$$ & & & & & & NS & 0.0207 \\
\hline \multicolumn{8}{|c|}{ IL-1RA/IL-1 $\alpha(\mathrm{pg} / \mu \mathrm{g})$} \\
\hline Treated & $293.5 \pm 301.2$ & $235.9 \pm 342.4$ & $-19.6^{*}$ & $241.9 \pm 392.2$ & $102.9 \pm 111.0$ & $-57.5^{*}$ & $-66.8^{* *}$ \\
\hline Control & & & & $230.2 \pm 294.7$ & $299.6 \pm 352.9$ & 30.1 & 7.9 \\
\hline$p$ value ${ }^{\$}$ & & & & & & 0.067 & 0.0211 \\
\hline \multicolumn{8}{|c|}{ Plakoglobin (arbitrary units) } \\
\hline Treated & $0.936 \pm 0.983$ & $0.546 \pm 0.712$ & $-41.7^{* *}$ & $0.401 \pm 0.388$ & $0.328 \pm 0.129$ & -18.2 & $-56.3^{*}$ \\
\hline Control & & & & $0.624 \pm 0.842$ & $0.650 \pm 0.275$ & $+4.2^{* *}$ & -39.7 \\
\hline$p$ value $\$$ & & & & & & 0.0095 & NS \\
\hline
\end{tabular}

Comparisons versus W0 using Student's $t$ test, ${ }^{*} p<0.05,{ }^{* *} p<0.005,{ }^{* * *} p<0.001,{ }^{* * *} p<0.0001$

Bold values are statistically significant changes

$S D$ standard deviation, $I L$ interleukin

\$ Intergroup comparison using an analysis of covariance (ANCOVA)

\section{DISCUSSION}

The effect of short-term intensive treatment with the investigational product has been demonstrated microbiologically, clinically and biochemically: a three-times weekly application exhibited an antifungal action (changes in Malassezia spp.), an anti-inflammatory effect (changes in specific markers of inflammation and pruritus associated with clinical results for erythema and itching) and antidandruff efficacy (cohesion markers associated with clinical 
Table 4 a, b, c: Correlations between clinical parameters and microbiological and biochemical data

\begin{tabular}{|c|c|c|c|c|}
\hline Correlation between & Group & $r$ & $p^{*}$ & $\bar{n}$ \\
\hline \multicolumn{5}{|l|}{ (a) During the whole study (from W0 to W10) } \\
\hline Overall clinical score for dandruff and histamine & Treated & 0.42028 & 0.0149 & 33 \\
\hline Overall clinical score for dandruff and $\log M$. restricta & Treated & 0.38194 & 0.0310 & 32 \\
\hline Erythema and IL-1RA/IL- $1 \alpha$ & Treated & 0.63538 & 0.0035 & 19 \\
\hline Erythema and histamine & Treated & 0.38470 & 0.0271 & 33 \\
\hline Erythema and PLK & Treated & 0.76376 & 0.0274 & 8 \\
\hline Global efficacy SGA and histamine & Treated & -0.37528 & 0.0314 & 33 \\
\hline Global efficacy SGA and log Malassezia total & Control & -0.38696 & 0.0346 & 30 \\
\hline Correlation between & $r$ & $p^{*}$ & $n$ & \\
\hline \multicolumn{5}{|l|}{ (b) During the intensive treatment phase (from W0 to W2) } \\
\hline Overall clinical score for dandruff and IL-1RA/IL-1 $\alpha$ & 0.40287 & 0.0055 & 46 & \\
\hline Global efficacy SGA and IL-1RA/IL-1 $\alpha$ & -0.35780 & 0.0146 & 46 & \\
\hline Correlation between & Group & $r$ & $p^{*}$ & $n$ \\
\hline \multicolumn{5}{|l|}{ (c) During the maintenance phase (from W2 to W10) } \\
\hline Overall clinical score for dandruff and $\log M$. globosa & Treated & 0.35862 & 0.0438 & 32 \\
\hline Global efficacy SGA and histamine & Treated & -0.36098 & 0.0390 & 33 \\
\hline Overall clinical score for dandruff and IL-1RA/IL- $1 \alpha$ & Control & 0.61709 & 0.0083 & 17 \\
\hline Itching and IL-1RA/IL- $1 \alpha$ & Control & 0.49488 & 0.0434 & 17 \\
\hline Itching and $\log$ Malassezia total $(=$ globosa + restricta $)$ & Control & 0.40976 & 0.0245 & 30 \\
\hline
\end{tabular}

Only significant correlations are shown

$P L K$ plakoglobin, $I L$ interleukin, $S G A$ subjective global assessment

* Spearman's test

results for scaling, including global dandruff score, self-assessment of dandruff). It should be noted that in this study, the intensive treatment phase was half as long as the standard intensive treatments, which usually last 4 weeks [25]). It is notable that effects were measured from the first application, which is of considerable interest to patients eager to be relieved of their symptoms and which might contribute to good compliance. This study was conducted during the winter, when the disease is more frequent but also when the symptoms are milder, requiring a much larger sample size to demonstrate a real treatment effect by the study product [26]. In our study, an effect was demonstrated despite a relatively small sample size $(n=33$ and $n=34$ for the treated and control groups, respectively). This effect declined after the discontinuation of treatment in the control group, but for some parameters a complete return to baseline was not observed, from which it may be concluded that some improvements persisted for 8 weeks after stopping treatment: overall clinical 
dandruff score, investigator's SGA, itching, IL-8 levels. This persistence shows the benefit of intensive treatment. With maintenance treatment (once a week), some improvements observed after the intensive treatment phase, such as $\log$ M. globosa, declined, but were still significant at W10 compared with baseline, while other improvements were maintained, i.e., Malassezia populations, irritation (erythema score), investigator's SGA score, selfassessment of scaling (dandruff), discomfort, itching, IL-8 level, cathepsin S level, and PLK. Other improvements were amplified, e.g., overall clinical dandruff score and IL-1RA/IL$1 \alpha$ ratio, whereas only one factor returned to baseline: histamine level.

A positive relationship was found between the main clinical signs of SD (dandruff, itching, and erythema) and the following biomarkers: Malassezia populations, histamine level, and IL1RA/IL-1 $\alpha$ ratio. The overall efficacy was negatively correlated with these biomarkers. In the case of cathepsin S, its levels decreased markedly at the end of the intensive treatment phase (by 70\%, $p<0.0001$ ).

Levels of cathepsin $S$, an activator of proteinase-activated receptor-2 (PAR2) [27], were recently shown to be increased, along with PAR2 and histamine, in subjects with SD, and to be correlated with clinical parameters linked to the severity of SD and itching, suggesting that cathepsin $\mathrm{S}$ could be used as a biomarker of pruritus. Biomarkers of pruritus [28], inflammation, hyperproliferation and skin barrier function were recently shown to be modified in SD and restored by therapeutic intervention [for review, see 4]. They can be studied by non-invasive sampling, enabling them to be used in routine clinical evaluations either as surrogate endpoints or as complementary ones to signs and symptoms. These new methods of biomolecular analysis allow a more comprehensive and objective evaluation of improvements during treatment.

So far, only a few recent studies have evaluated SD treatment by criteria other than clinical and microbiological [10, 19, 24]. Mills et al. [19] performed a systematic study of the transcriptome of biopsies of SD scalp lesions. They showed that SD was characterized by a diminished expression of genes involved in lipid metabolism and an increased expression of genes involved in inflammation and that, under conditions in which symptoms resolved, ZP produced a transcriptomic profile similar to healthy skin. However, this method of analysis cannot be easily applied in large-scale clinical studies.

A non-invasive skin surface sampling methodology combined with highly sensitive ELISA-based quantification was developed in 2001 [29], permitting larger clinical sample sizes. This methodology was applied to differentiate SD from normal subjects and showed a link between classical criteria of SD evaluation and IL-1RA (ratio to IL-1 $\alpha$ ), IL-2, IFN$\gamma$, nitric oxide and TNF- $\alpha$ [30]. Recently, Kerr et al. [24] compared normal and SD subjects before and after a 3-week treatment with a ZPcontaining shampoo and showed an association between the subjective perception of itch in the scalp and histamine level in the SC, both before and after treatment. Kerr et al. [10] further investigated a set of biomarkers in subjects with dandruff treated for 3 weeks with a commercial ZP shampoo versus a control. They showed that the resolution of flaking with treatment was associated with a reduction in inflammatory biomarkers (IL-1 $\alpha$, IL-1RA, IL-8), and a dramatic improvement in biomarkers of epidermal barrier integrity (keratin 1, 10, 11; involucrin; SC lipids; human serum albumin). This combination of biomarkers appeared to provide a good picture of the condition of the 
scalp in SD. This was the first demonstration of a set of tools, other than flaking scores, that allows an objective assessment of scalp treatment.

In 2013, Schwartz et al. [4] reviewed the structural and biomolecular abnormalities characterizing SD. They concluded that biomarkers representing inflammation, hyperproliferation and barrier function were all perturbed by the SD condition and robustly responded to therapeutic resolution. A number of biomarkers were found to be significantly correlated to the key SD symptom of flaking, such as IL-1RA: IL-1 $\alpha$, IL-8, histamine, involucrin, keratins.

Our study using other biomarkers, especially cathepsin and a method of clinical scoring, focusing on erythema and pruritus, rather than on desquamation, provides a new insight on the use of biomarkers as new clinical measurement endpoints in SD clinical trials. The use of biomarkers in medical product evaluation has been recommended by the U.S. Food and Drug Administration (FDA) in a recent white paper "Innovation or Stagnation?" [31]. Moreover, Schwartz et al. [4], argued that since biomarkers reflect disease mechanisms underlying the clinical pathophysiology, they could be useful tools fort early detection of the condition.

Based on the inflammatory component of $\mathrm{SD}$, the lack of synchronization between proliferation and differentiation, and the impaired barrier function, Schwartz et al. suggested that SD could belong to the group of inflammatory dermatoses, including psoriasis, atopic dermatitis and acne, although these conditions differ in their triggering events [4]. Accordingly, the addition of 18ßGA, shown to exert an anti-inflammatory action in cultured cells [22, 23] and in a model of acute inflammation in the mouse ear [22], could contribute to the in vivo anti-inflammatory action observed in our study, in combination with CPO and ZP.

In conclusion, the effectiveness of this investigational product on the maintenance phase of SD was clearly and objectively demonstrated at the clinical, biochemical and microbiological levels: the decrease in biomarkers and symptoms of SD persisted at 10 weeks in the treated group. In line with FDA recommendations encouraging the use of biomarkers [31], the correlations observed in our study between clinical signs and biomarkers provide clues to explain the resolution of SD at the molecular level and confirm the value of biomolecular endpoints for the detailed assessment of SD treatment. The fact that our methodology allows biomarkers to be studied through non-invasive sampling is particularly relevant on both practical and ethical grounds. Furthermore, the convergence of conventional clinical measurements and objective measurements strongly demonstrates the usefulness of maintenance therapy.

\section{ACKNOWLEDGMENTS}

Editorial assistance in the preparation of this manuscript was provided by Drs. Françoise Nourrit-Poirette and Marielle Romet, from Santé Active Edition (Bois-Colombes, France) Support for this assistance was funded by Pierre Fabre Dermo-Cosmétique (Boulogne Billancourt, France). This study was supported by Pierre Fabre Dermo-Cosmétique. The sponsor provided funding for technical (including products and data analysis), editorial assistance and article processing charges but had no role in the design and conduct of the study, in the collection, analysis, and interpretation of data, or in the preparation of the manuscript, review, or approval of the 
manuscript. All named authors meet the ICMJE criteria for authorship for this manuscript, take responsibility for the integrity of the work as a whole, and have given final approval for the version to be published.

Conflict of interest. V. Turlier, C. Viode, A. Bacquey, O. Lejeune, C. Lauze, C. Villeneuve, A. Rouquier, C. Casas, D. Redoules, V. Mengeaud, A.-M. Schmitt are employed by Pierre Fabre Laboratories and received salaries, but they do not have any financial interests in the findings described in this manuscript. E. Durbize and R. Oliveira Soarres are private practitioners, who received fees for their role as investigators in this study.

Compliance with ethics. All procedures were in accordance with the ethical principles stated in the Declaration of Helsinki of 1975, as revised in 2000 and 2008, in conformity with local legal requirements in each country and after approval of the study protocol by the Lisbon Hospital Ethic Committee. Informed consent was obtained from all patients before being included in the study.

Open Access. This article is distributed under the terms of the Creative Commons Attribution Noncommercial License which permits any noncommercial use, distribution, and reproduction in any medium, provided the original author(s) and the source are credited.

\section{REFERENCES}

1. Johnson M-LT, Roberts J. Prevalence of dermatological diseases among persons 1-74 years of age: United States. Advance data Report No. 4. U.S. Department of Health, Education, and Welfare. 1977. http://www.cdc.gov/nchs/data/series/sr_11/ sr11_212.pdf. Last Accessed 1 June 2013.
2. Foley P, Zuo Y, Plunkett A, Merlin K, Marks R. The frequency of common skin conditions in preschoolaged children in Australia: seborrheic dermatitis and pityriasis capitis (cradle cap). Arch Dermatol. 2003;139:318-22.

3. Breunig Jde A, De Almeida HL Jr, Duquia RP, Souza PR, Staub HL. Scalp seborrheic dermatitis: prevalence and associated factors in male adolescents. Int J Dermatol. 2012;51:46-9.

4. Schwartz JR, Messenger AG, Tosti A, et al. A comprehensive pathophysiology of dandruff and seborrheic dermatitis-towards a more precise definition of scalp health. Acta Derm Venereol. 2013;93:131-7.

5. Jang SJ, Lim SH, Ko JH, et al. The investigation on the distribution of Malassezia yeasts on the normal Korean skin by $26 \mathrm{~S}$ rDNA PCR-RFLP. Ann Dermatol. 2009;21:18-26.

6. Sandström Falk M-H, Tengvall Linder M, Johansson $\mathrm{C}$, et al. The prevalence of Malassezia yeasts in patients with atopic dermatitis, seborrhoeic dermatitis and healthy controls. Acta Derm Venereol. 2005;85:17-23.

7. Tajima M, Sugita T, Nishikawa A, Tsuboi R. Molecular analysis of Malassezia microflora in seborrheic dermatitis patients: comparison with other diseases and healthy subjects. J Invest Dermatol. 2008;128:345-51.

8. Oh BH, Lee YW, Choe YB, Ahn KJ. Epidemiologic study of Malassezia yeasts in seborrheic dermatitis patients by the analysis of $26 \mathrm{~S}$ rDNA PCR-RFLP. Ann Dermatol. 2010;22:149-55.

9. Zhang H, Ran Y, Xie Z, Zhang R. Identification of Malassezia species in patients with seborrheic dermatitis in China. Mycopathologia. 2013;175:83-9.

10. Kerr K, Darcy T, Henry J, et al. Epidermal changes associated with symptomatic resolution of dandruff: biomarkers of scalp health. Int J Dermatol. 2011;50:102-13.

11. Squire RA, Goode K. A randomised, single-blind, single-centre clinical trial to evaluate comparative clinical efficacy of shampoos containing ciclopirox olamine $(1.5 \%)$ and salicylic acid (3\%), or ketoconazole $(2 \%$, Nizoral) for the treatment of dandruff/seborrhoeic dermatitis. J Dermatolog Treat. 2002;13:51-60.

12. Faergemann J. Seborrhoeic dermatitis and Pityrosporum orbiculare: treatment of seborrhoeic dermatitis of the scalp with miconazolehydrocortisone (Daktacort), miconazole and hydrocortisone. Br J Dermatol. 1986;114:695-700. 
13. Saple DG, Ravichandran G, Desai A. Evaluation of safety and efficacy of ketoconazole $2 \%$ and zinc pyrithione $1 \%$ shampoo in patients with moderate to severe dandruff-a postmarketing study. J Indian Med Assoc. 2000;98:810-1.

14. Lassus A, Nolting KS, Savopoulos C. Comparison of ciclopirox olamine $1 \%$ cream with ciclopirox $1 \%$ hydrocortisone acetate $1 \%$ cream in the treatment of inflamed superficial mycoses. Clin Ther. 1988;10:594-9.

15. Rosen T, Schell BJ, Orengo I. Anti-inflammatory activity of antifungal preparations. Int J Dermatol. 1997;36:788-92.

16. Van Cutsem J, Van Gerven F, Fransen J, Schrooten $\mathrm{P}$, Janssen PA. The in vitro antifungal activity of ketoconazole, zinc pyrithione, and selenium sulfide against Pityrosporum and their efficacy as a shampoo in the treatment of experimental pityrosporosis in guinea pigs. J Am Acad Dermatol. 1990;22:993-8.

17. McGinley KJ, Leyden JJ. Antifungal activity of dermatological shampoos. Arch Dermatol Res. 1982;272:339-42.

18. Warner RR, Schwartz JR, Boissy Y, Dawson TL Jr. Dandruff has an altered stratum corneum ultrastructure that is improved with zinc pyrithione shampoo. J Am Acad Dermatol. 2001;45:897-903.

19. Mills KJ, Hu P, Henry J, Tamura M, Tiesman JP, Xu J. Dandruff/seborrhoeic dermatitis is characterized by an inflammatory genomic signature and possible immune dysfunction: transcriptional analysis of the condition and treatment effects of zinc pyrithione. Br J Dermatol. 2012;166:S33-40.

20. Roques C, Brousse S, Panizzutti C. In vitro antifungal efficacy of ciclopirox olamine alone and associated with zinc pyrithione compared to ketoconazole against Malassezia globosa and Malassezia restricta reference strains. Mycopathologia. 2006;162:395-400.

21. Lorette G, Ermosilla V. Clinical efficacy of a new ciclopirox olamine/zinc pyrithione shampoo in scalp seborrheic dermatitis treatment. Eur J Dermatol. 2006;16:558-64.

22. Ishida $\mathrm{T}$, Mizushina $\mathrm{Y}$, Yagi $\mathrm{S}$, et al. Inhibitory effects of glycyrrhetinic acid on DNA polymerase and inflammatory activities. Evid Based Complement Alternat Med. 2012;2012:650514.

23. Wang CY, Kao TC, Lo WH, Yen GC. Glycyrrhizic acid and 18ß-glycyrrhetinic acid modulate lipopolysaccharide-induced inflammatory response

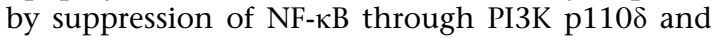
p110 $\gamma$ inhibitions. J Agric Food Chem. 2011;59:7726-33.

24. Kerr K, Schwartz JR, Filloon T, et al. Scalp stratum corneum histamine levels: novel sampling method reveals association with itch resolution in dandruff/ seborrhoeic dermatitis treatment. Acta Derm Venereol. 2011;91:404-8.

25. Shin $\mathrm{H}$, Kwon OS, Won $\mathrm{CH}$, et al. Clinical efficacies of topical agents for the treatment of seborrheic dermatitis of the scalp: a comparative study. J Dermatol. 2009;36:131-7.

26. Weiss SC, Rowell R, Krochmal L. Impact of seasonality on conducting clinical studies in dermatology. Clin Dermatol. 2008;26:565-9.

27. Reddy VB, Shimada SG, Sikand P, Lamotte RH, Lerner EA. Cathepsin S elicits itch and signals via protease-activated receptors. J Invest Dermatol. 2010;130:1468-70.

28. Viode C, Lejeune O, Villeneuve C, et al. Evidence of cathepsin S, PAR2 and histamine interest in pruritus aspect of clinical dandruff/seborrheic dermatitis evaluation-poster $\mathrm{n}^{\circ}$ 107. Acta Dermatol Venereol. 2013;93:624.

29. Perkins MA, Osterhues MA, Farage MA, Robinson MK. A noninvasive method to assess skin irritation and compromised skin conditions using simple tape adsorption of molecular markers of inflammation. Skin Res Technol. 2001;7:227-37.

30. Perkins M, Cardin C, Osterhues M, Robinson M. A non-invasive tape absorption method for recovery of inflammatory mediators to differentiate normal from compromised scalp conditions. Skin Res Technol. 2002;8:187-93.

31. US Department of Health and Human Services FDA. Innovation or stagnation? Challenge and opportunity on the critical path to new medical products. 2004; http://www.fda.gov/Science Research/SpecialTopics/CriticalPathInitiative/Critical PathOpportunitiesReports/ucm077262.htm. Last Accessed 1 June 2013. 